\title{
3D Facial Analysis Software for Before and After Comparison
}

\author{
Masaki HAYASHI ${ }^{a, b}$, Kohei IKE ${ }^{a}$, Yuji NISHIO ${ }^{a}$, Hideto KAMESHIMA ${ }^{a}$, Yukio SATO ${ }^{a, b}$ \\ a Spacevision, Tokyo, Japan; \\ ${ }^{\mathrm{b}}$ Keio University, Yokohama, Japan \\ http://dx.doi.org/10.15221/13.352
}

\begin{abstract}
Spacevision developed a software which can align two 3D faces automatically by a machine learning technique. With automatically aligned two face data, the software provides 3D comparison techniques between before/after faces, such as heat-map visualization, cross-section and the other geometric measurements. We believe the software will open up a new application for 3D face scanners in especially beauty salons and aesthetic shops.
\end{abstract}

Keywords: 3D face scanning, 3D face data comparison, 3D registration, before and after.

\section{Introduction}

Comparison between two data is one of the key components of 3D face scanning for 3D face scanners to become widespread in many retailers, especially for beauty and healthcare industries, who need before and after analysis to show the effect of their services to the customers. For example, in cosmetics, visualizing two face data itself is quite essential to show the customers the faces before and after the makeup.

In cosmetic surgery, however, visualization is not enough. Difference in $3 D$ Shape does matter. In this sense, some automatic comparison schemes should be provided with 3D face scanners in order to predict/simulate the result of a surgery and to easily confirm the actual result of the surgery. 3D "before and after" shape analysis is also critical for beauty salons because they provide many types of manual and machinery massage which try to make the customer's shape thinner and healthier.

To provide the before and after comparison for those shops and salons, Spacevision developed a new 3D face analysis software (see figure 1). Firstly, the software reads any types of 3D frontal facial point clouds of an identical person. Then the software automatically aligns the point clouds to the canonical same direction with landmark detection (nose tip) and PCA (principal component analysis). Finally, the software can perform several kinds of 3D shape comparison between two point clouds, such as heat-map visualization, cross-section and the other geometric measurements.

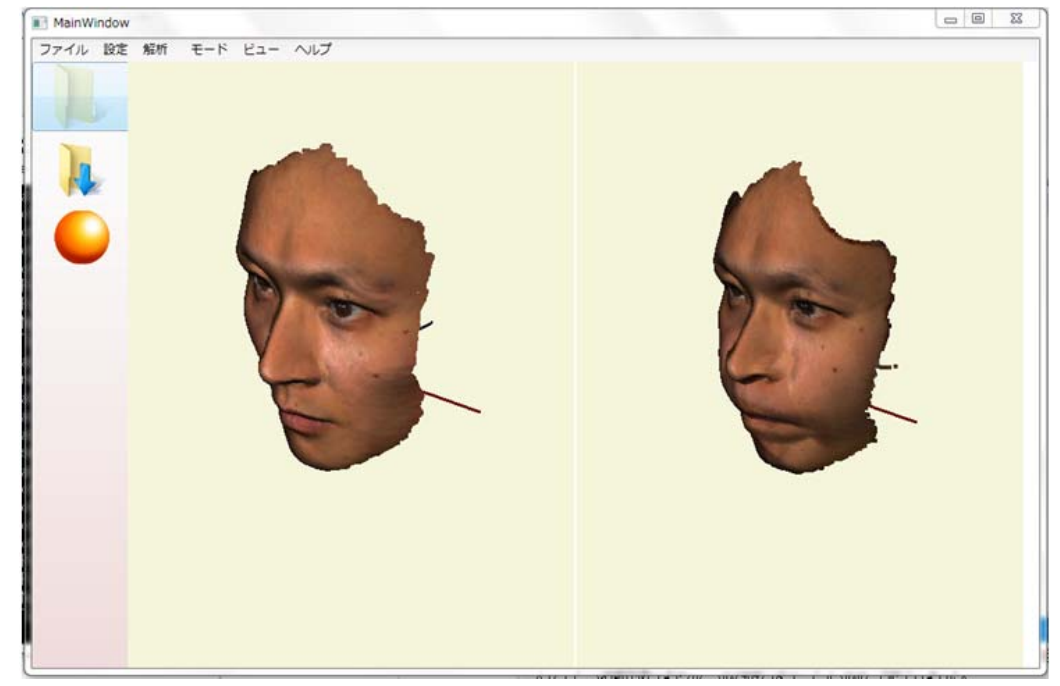

Fig. 1. Spacevision's facial analysis software. The main feature is comparison between two facial point clouds via automatic alignment.

\footnotetext{
* hayashi@space-vision.jp; +81- 3- 5428-4144; www.space-vision.jp
} 


\section{Face alignment}

Our alignment strategy is based on the alignment part of automatic 3D face recognition method of [1]. The method firstly detects the nose tip from the input point cloud by fitting a line model to the nose-tip candidates extracted from each vertical section. Next, the nose tip is used to crop the input face data. By performing PCA twice with the cropped face, the original face data is finally aligned to the canonical direction and position.

This alignment scheme of [1] is robust to facial expressions because it utilizes the principal data direction of the cropped facial data with two sizes (whole face region and nose region). Hence, the software achieves automatic alignment function even when the facial expression occurs between two data of the same person.

\section{Face Comparison}

\subsection{Heat-map Analysis}

Our software offers heat-map visualization of the distances against the other facial surface (see figure 2). With two facial point clouds aligned by the method in Section.2, the software calculates the minimum distance to the other point cloud surface at every point. This provides the user an intuitive visualization as a heat map to know the magnitude of difference between the before and after data at each local region.

Note that "facial model fitting" is the better way to calculate more dense and accurate point-to-point matching between the facial surfaces. Also, the model fitting makes it possible to the large-scale statistical analysis from 3D human body database. Hence, we are planning to provide a model fitting to a facial 3D model in the newer version of the software.

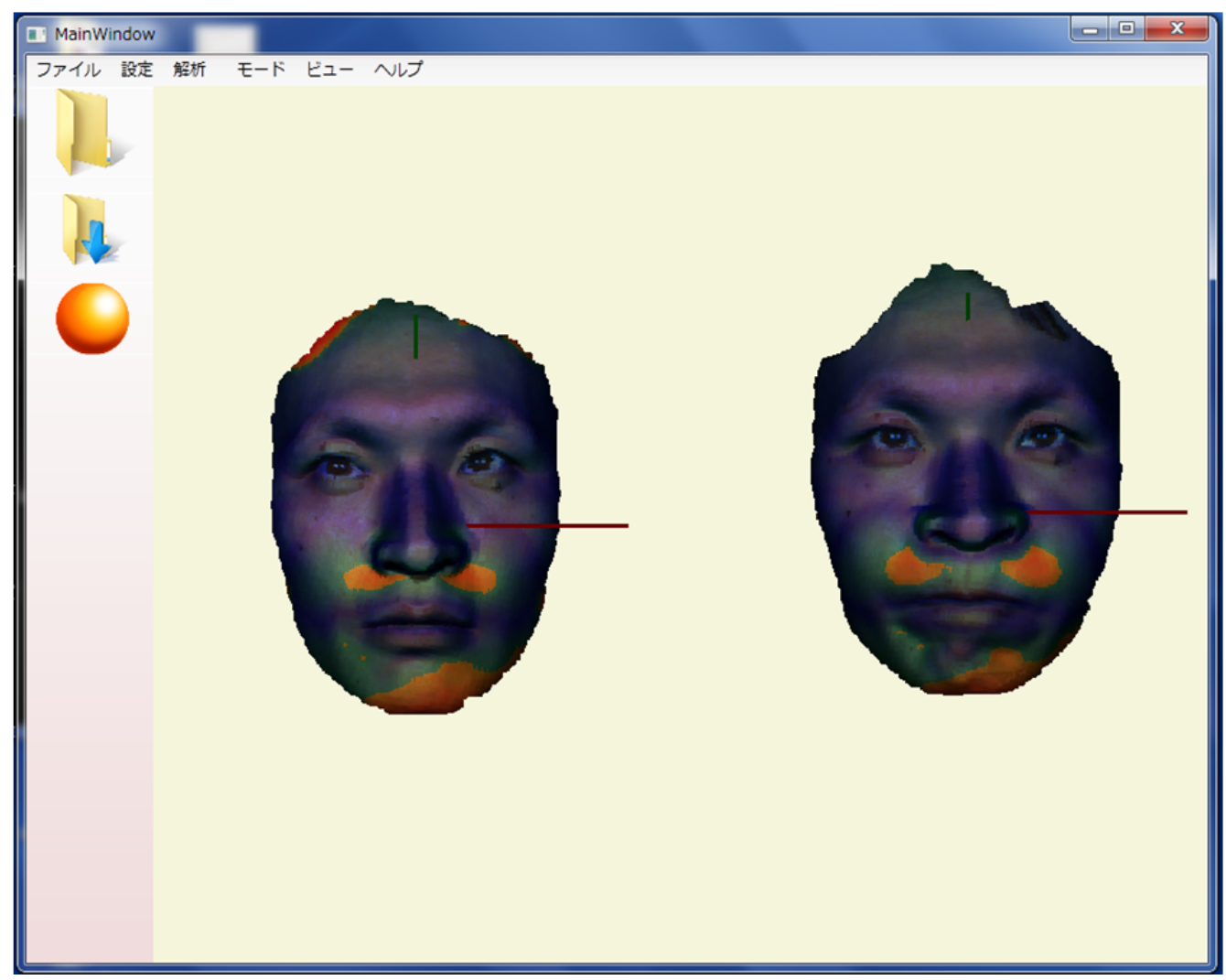

Fig. 2. Heat-Map visualization of two faces. 


\subsection{Cross Section Comparison}

The software also provides the comparison of cross sections between two facial data (see figure 3 ). While the software only detects nose tip as facial landmarks, the PCA-based alignment approach [1] detects the center of a facial point cloud robustly. Therefore, visualization of aligned two cross sections (vertical/horizontal) is quite useful for comparing the vertical or horizontal difference.

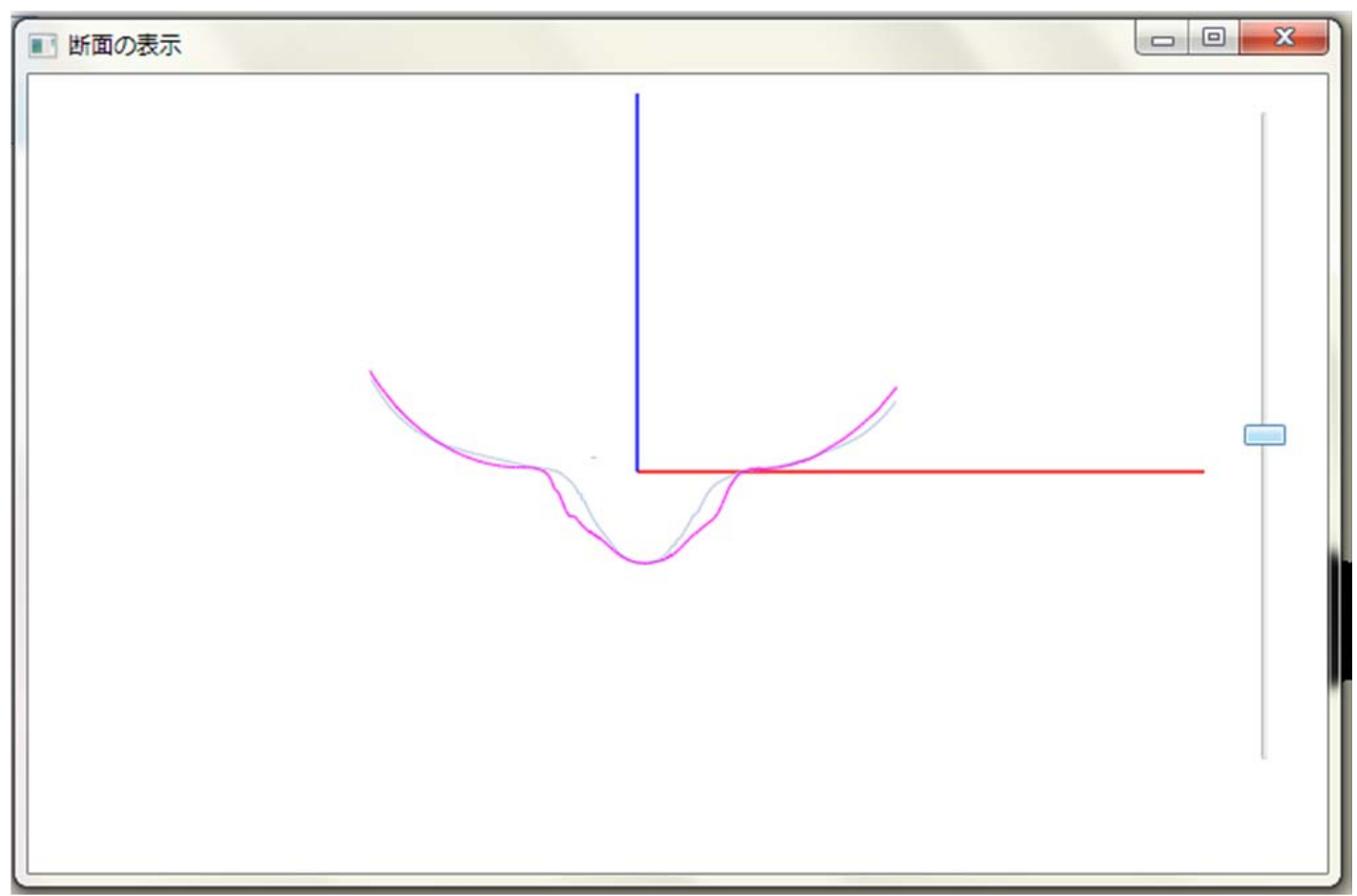

Fig. 3. Comparison of cross sections. Two contours are the vertical section of each data at the same height.

\section{Conclusion}

Spacevision developed a new software for automatic 3D facial data comparison. The automatic alignment and comparison schemes realizes objective and fair way to measure and compare the before and after facial data.

As future plans, we will provide automatic detection of standard facial landmarks (eyes, nose and mouth) and the face model fitting to achieve statistical face analysis and facial data simulation for computer graphics.

\section{References}

1. Mian, Ajmal S., Mohammed Bennamoun, and Robyn Owens. "An efficient multimodal 2D-3D hybrid approach to automatic face recognition." Pattern Analysis and Machine Intelligence, IEEE Transactions on 29.11 (2007): 1927-1943. 